\title{
LA RELACIÓN ENTRE LA INTELIGENCIA EMOCIONAL Y LA PERSONALIDAD EN ESTUDIANTES DE EDUCACIÓN SECUNDARIA
}

\author{
Ana Parodi Úbeda \\ Universidad de Alicante, (ana.parodi@ua.es) \\ Víctor Belmonte Lillo \\ Universidad de Alicante, \\ Carmen Ferrándiz García \\ Universidad de Murcia \\ María José Ruiz Melero \\ Universidad de Murcia \\ https://doi.org/10.17060/ijodaep.2017.n1.v2.926
}

Fecha de Recepción: 7 Marzo 2017

Fecha de Admisión: 1 Abril 2017

\section{RESUMEN}

La investigación se centra en analizar las relaciones entre personalidad, desde el modelo Big Five (Caprara, Barbaranelli \& Borgogni, 1993), y la Inteligencia Emocional Autopercibida (IEA) en alumnado de Educación Secundaria Obligatoria (ESO). La muestra estuvo compuesta por un total de 670 adolescentes con edades comprendidas entre los 12 y los 16 años $(M=13.86 ; D T=1.232)$. La IEA aglutina los modelos denominados mixtos: la Competencia Socioemocional (BarOn \& Parker, 2000) valorada mediante el EQ-i:YV; y la Autoeficacia Emocional (Petrides, Sangareau, Furnham \& Frederickson, 2006) medida con el TEIQue-ASF. Los rasgos de personalidad se evaluaron mediante BFQ-NA (adaptación española de Del Barrio, Carrasco \& Holgado, 2006). Nuestros resultados evidencian la estrecha relación entre la Autoeficacia Emocional y los rasgos de personalidad del modelo de Cinco Grandes, en consonancia con la teoría del constructo que indica que este tipo inteligencia emocional se compone de una disposición de rasgos y habilidades emocionales, situadas en los niveles más bajos de la estructura de la personalidad. Asimismo, se constatan relaciones significativas entre el modelo de Competencia Socio-emocionaly los rasgos del modelo de los Cinco Grandes.

Palabras clave: Competencia socio-emocional, personalidad, Inteligencia Emocional Autopercibida

\section{ABSTRACT}

The research focuses on the relationships between personality, from the Big Five model (Caprara, Barbaranelli \& Borgogni, 1993), and Self-perceived Emotional Intelligence (IEA) in sec- 


\section{LA RELACIÓN ENTRE LA INTELIGENCIA EMOCIONAL Y LA PERSONALIDAD EN ESTUDIANTES DE EDUCACIÓN SECUNDARIA}

ondary education (ESO) students. The sample consisted of a total of 670 adolescents between the ages of 12 and $16(M=13.86 ; D T=1232)$. The IEA agglutinates the models designated mixed: the Socio-emotional Competence (BarOn\& Parker, 2000) valued by the EQ-i: YV; And Emotional SelfEfficacy (Petrides, Sangareau, Furnham\& Frederickson, 2006) as measured by TEIQue-ASF. Personality traits were assessed using BFQ-NA (Spanish adaptation of Del Barrio, Carrasco \&Holgado, 2006). Our results evidence the close relationship between Emotional Self-efficacy and personality traits of the Big Five model, in line with the construct theory that indicates that this type of emotional intelligence is composed of an array of emotional traits and abilities, Lower personality structure. Likewise, significant relationships between the Socio-Emotional Competence model and the features of the Big Five model are found.

Keywords: Socio-emotional competence, personality, Self-perceived Emotional Intelligence

\section{ANTECEDENTES}

Con bastante frecuencia para explicar cómo y por qué nos comportamos de determinado modo en las distintas situaciones y momentos temporales de nuestra vida solemos hacer referencia a nuestros sentimientos y estados de ánimo. Desde que el concepto de Inteligencia Emocional se diera a conocer de manera científica hace ya más de veinte años, se ha convertido en un emocionante y productivo campo de investigación, siendo uno de los debates más discutidos su relación con determinados rasgos de la personalidad. Los diversos estudios en este sentido evidencian resultados poco concluyentes. La propia terminología en la que se han planteado las distintas concepciones de Ia Inteligencia Emocional aproxima inevitablemente este concepto a los factores que definen la personalidad de los individuos.

Inicialmente, la Inteligencia Emocional se plantea como una habilidad, asumiendo el marco teórico y metodológico propio de la tradición psicométrica, de manera que ésta es concebida como una capacidad, independiente de la personalidad, susceptible de ser aprendida o modificada y que evoluciona a lo largo del desarrollo cognitivo y madurativo de los sujetos. Posteriormente, enfoques alternativos presuponen que las habilidades que comprende este concepto aparecen, por lo general, en individuos con determinadas características personales. Esta asunción da lugar a los denominados modelos mixtos, en los que el concepto de Inteligencia Emocional es reformulado y presentado como una combinación de capacidades, destrezas y rasgos de personalidad. Incluido dentro de estos modelos es donde uno de sus autores más relevantes, Petrides, acuña el término de Autoeficacia Emocional, en cuyo marco la Inteligencia Emocional se conceptualiza como un rasgo de personalidad, localizado en los niveles inferiores de las jerarquías de la personalidad.

Parece ser que lo que conduce a constructos diferentes de Inteligencia Emocional (como una inteligencia más o como parte de la personalidad), no son los modelos ni los elementos que los integran, ya que éstos tienden a ser complementarios más que contradictorios entre sí, sino la ejecución y el método de medida empleados: por una parte, dicha ejecución o la manera de hacer operativo el concepto de la Inteligencia Emocional es entendida como una capacidad cognitiva que conduce a un constructo diferente del que se deriva de su realización como un rasgo de personalidad (Pérez, Petrides \& Furnham, 2005); por otra parte, cada uno de estos planteamientos teóricos ha evolucionado de forma paralela a la construcción de sus correspondientes instrumentos de medida. El modelo de Inteligencia Emocional como habilidad ha desarrollado pruebas de ejecución en las que el nivel de competencia es presumiblemente independiente de las características personales de los individuos, mientras que los modelos de Inteligencia Emocional Autopercibida, que acoge a los modelos mixtos, se sirven de medidas de auto-informe (como las empleadas para medir la personalidad), al entender que ésta es una forma más apropiada de estimar la Inteligencia Emocional. 
Los actuales modelos de la personalidad tienen como punto de partida las teorías de Eysenck (1952), quien propone dos principios fundamentales para su estudio: la aplicación de métodos factoriales a la búsqueda de rasgos y la vinculación de los rasgos observables con sus sustratos biológicos. Posteriormente, estos dos principios han desembocado en dos corrientes de investigación: por un lado, el Modelo de los Cinco Grandes Factores de la Personalidad (Costa \& McCrae, 1992) parte del estudio factorial de los rasgos de personalidad obtenidos a partir de auto-informes basados en categorías presentes en el léxico habitual de las personas, sin proponer explícitamente ningún sustrato biológico para los factores encontrados; y por otro, en el polo opuesto, Cloninger (1987) quien formula una serie de rasgos temperamentales teóricamente vinculados a los sistemas de neurotransmisión cerebral, así como otros rasgos caracteriales, vinculados al historial de aprendizaje.

\section{OBJETIVO}

Estudiar la relación entre la IE Autopercibida (basada en los modelos mixtos, valorada a través de los cuestionarios TEIQue-ASF y EQ-i:YV) y la personalidad (basada en el modelo de "los Cinco Grandes" y valorada con el BFQ-NA).

\section{MUESTRA}

La investigación se lleva a cabo con una muestra compuesta por 670 alumnos (46.6\% varones) estudiantes de $1^{\circ}(31.9 \%)$, 2ำ $(26.9 \%)$, 3ำ $(23.6 \%)$ y $4^{\circ}(17.6 \%)$ de Educación Secundaria Obligatoria (ESO) pertenecientes a diferentes Centros privados-concertados de la provincia de Alicante, con edades comprendidas entre los 12 y los 16 años $(M=13.86 ; D T=1.232)$. La selección de los participantes se realiza mediante un muestreo de carácter incidental.

\section{METODOLOGÍA E INSTRUMENTOS}

Los instrumentos empleados para alcanzar los objetivos de nuestro estudio son:

- BFQ-NA, Cuestionario 'Big Five' de personalidad para niños y adolescentes (Barbaranelli, Caprara y Rabasca, 1998; Adaptación española: Del Barrio, Carrasco y Holagado, 2006). Representa la adaptación del modelo de los Cinco Grandes de la personalidad para niños y adolescentes. Comprende cinco dimensiones: Conciencia (evalúa autonomía, orden, precisión y el cumplimiento de normas y compromisos), Apertura (incluye elementos de aspectos intelectuales, creatividad, intereses culturales), Extraversión (actividad, entusiasmo, asertividad y autoconfianza), Amabilidad (preocupación y sensibilidad hacia los otros y sus necesidades) e Inestabilidad emocional (ansiedad, depresión, descontento 0 ira). El ámbito de aplicación abarca edades entre 8 y 15 años. El cuestionario consta de 65 elementos de respuesta múltiple (tipo Likert: de $1=$ Casi siempre a $5=$ Casi nunca).

- Cuestionario de Inteligencia Emocional: Versión para Adolescentes, EQ-i YV (Bar-On y Parker, 2000). Es un instrumento que proporciona información sobre la percepción que un sujeto tiene sobre sus competencias emocionales y sociales, permitiendo trazar un perfil social y afectivo. Está destinado a alumnos de edades comprendidas entre 7 y 18 años, y consta de 60 afirmaciones en las que valoran las siguientes dimensiones: Intrapersonal, Interpersonal, Manejo del Estrés, Adaptabilidad y Estado de Ánimo general (el rango de la escala oscila desde 1=Nunca me pasa y 4=Siempre me pasa).

- Cuestionario de Inteligencia Emocional Rasgo Reducido para Adolescentes V.02, TEIQue-ASF (Petrides, Sangareau, Furnham y Frederickson, 2006) en su adaptación al castellano (Ferrando y Serna, 2006). Se trata de una versión simplificada en términos de complejidad sintáctica y léxica de la versión reducida para adultos (TEIQue-SF). Está destinada a alumnos-as de entre 12 a 17 años. 


\section{LA RELACIÓN ENTRE LA INTELIGENCIA EMOCIONAL Y LA PERSONALIDAD EN ESTUDIANTES DE EDUCACIÓN SECUNDARIA}

Compuesto de 30 ítems en escala Likert de 7 puntos, permite medir quince subescalas que dan cuenta de cuatro factores: bienestar emocional, autocontrol emocional, emocionabilidad y sociabilidad.

Para analizar los datos se aplicaron diferentes procedimientos:

- Para el análisis de fiabilidad de los instrumentos se utilizó el coeficiente de consistencia interna: Alfa de Cronbach.

- El análisis de correlación para estudiar la relación entre las dimensiones de la IE, la personalidad, la inteligencia y el rendimiento académico definidas en nuestro estudio, fue realizado mediante el coeficiente de correlación de Pearson.

Estos análisis se realizaron mediante el empleo del programa informatizado de análisis estadístico SPSS v.19.0 para Mac 0s.

\section{RESULTADOS}

\section{Análisis de fiabilidad de los instrumentos empleados}

Todos instrumentos utilizados muestran una adecuada fiabilidad. Los resultados obtenidos manifiestan que: el EQ-i: YV muestra una consistencia interna para el total de la escala de $=.89$. Es adecuada también la fiabilidad de las subescalas del cuestionario: Estado de ánimo ( = .88), Adaptabilidad ( = .83), Manejo del Estrés ( = .82), Interpesonal $=.76$ ) e Intrapersonal ( = .79); el TEIQue-ASF muestra $=.79$ también adecuada para su uso. Por su parte, el BFQ-NA reporta una fiabilidad $=.858$.

\section{Análisis correlacional entre IE autopercibida y factores del BFQ-NA.}

La Tabla 1 muestra los coeficientes de correlación de Pearson entre las variables edad, dimensiones de la escala de Competencia Socio-emocional de Bar-On, la puntuación total de la escala de Autoeficacia Emocional TEIQue-ASF y los rasgos de personalidad valorados en la prueba de personalidad BFQ-NA.

Tabla 1.

Correlaciones entre variables: IE, edad y personalidad

\begin{tabular}{|c|c|c|c|c|c|c|c|c|c|c|c|c|}
\hline & 1 & 2 & 3 & 4 & 5 & 6 & 7 & 8 & 9 & 10 & 11 & 12 \\
\hline 1. Edad & 1 & & & & & & & & & & & \\
\hline 2. Estado Ánimo & $-.102^{*}$ & 1 & & & & & & & & & & \\
\hline 3. Adaptabilidad & -.016 & $.472^{* *}$ & 1 & & & & & & & & & \\
\hline 4. Manejo Estrés & -.054 & $.250^{* *}$ & $.210^{* *}$ & 1 & & & & & & & & \\
\hline 5. Interpersonal & .046 & $.324^{* *}$ & $.406^{* *}$ & $.236^{* *}$ & 1 & & & & & & & \\
\hline 6. Intrapersonal & .050 & $.278^{* *}$ & $.235^{* *}$ & $.124^{* *}$ & $.214^{* *}$ & 1 & & & & & & \\
\hline $\begin{array}{l}\text { 7. Autoeficacia } \\
\text { Emocional }\end{array}$ & -.038 & $.626^{* *}$ & $.366^{* *}$ & $.472^{* *}$ & $.358^{* *}$ & $.250^{* *}$ & 1 & & & & & \\
\hline 8. Conciencia & $-.103^{* *}$ & $.336^{* *}$ & $.468^{* *}$ & $.365^{* *}$ & $.371^{* *}$ & $.144^{* *}$ & $.444^{* *}$ & 1 & & & & \\
\hline 9. Apertura & $-.094^{*}$ & $.395^{* *}$ & $.526^{* *}$ & $.312^{* *}$ & $.226^{* *}$ & $.137^{* *}$ & $.468^{* * *}$ & $.765^{* *}$ & 1 & & & \\
\hline 10. Extraversión & -.036 & $.393^{* *}$ & $.286^{* *}$ & .025 & $.399^{* *}$ & $.204^{* *}$ & $.347^{* *}$ & $.394^{* *}$ & $.290^{* *}$ & 1 & & \\
\hline 11.Amabilidad & .058 & $.292^{* *}$ & $.315^{* *}$ & $.291^{* *}$ & $.562^{* *}$ & $.287^{* *}$ & $.435^{* *}$ & $.586^{* *}$ & $.419^{* *}$ & $.485^{* *}$ & 1 & \\
\hline $\begin{array}{l}\text { 12. Inestabilidad } \\
\text { Emocional }\end{array}$ & .017 & $-.399^{* *}$ & $-.190^{* *}$ & $-.652^{* *}$ & $-.138^{* *}$ & $-.090^{*}$ & $-.523^{* *}$ & $-.214^{* *}$ & $-.217^{* *}$ & -.068 & $-.207^{* *}$ & 1 \\
\hline
\end{tabular}

* La correlación es significante al nivel 0.05 (bilateral).

** La correlación es significativa al nivel 0.01 (bilateral). 
En general, se observan relaciones significativas, de magnitud media y signo positivo entre las dimensiones de una misma escala entre sí. La edad mostró relaciones bajas y no significativas con la mayoría de variables a excepción del rasgo Conciencia que mostró relación estadísticamente significativa, aunque negativa y baja $(r=-.103 ; p<.001)$.

Con respecto a los rasgos de personalidad, las relaciones más elevadas se dieron con las dimensiones valoradas en las escalas de auto-informe (EQ:i-YV y TEIQue-ASF), entre ellas se destacan las relaciones entre Conciencia y Apertura con Adaptabilidad; así como Conciencia y Apertura con la puntuación total de la escala TEIQue-ASF. La dimensión de Inestabilidad Emocional mostró relaciones elevadas de signo negativo y estadísticamente significativas con la puntuación total del TEIQue-ASF.

\section{DISCUSIÓN Y CONCLUSIONES}

El presente estudio plantea si existe una relación entre la IE Autopercibida y la personalidad.

Se han analizado las características psicométricas referidas a fiabilidad y validez de los instrumentos (EQ-i: YV y BFQ-NA). Podemos afirmar que los instrumentos utilizados reúnen las características psicométricas adecuadas para su administración y uso, tal y como se esperaba.

El cuestionario de Autoeficacia Emocional (TEIQue-ASF) utilizado, presenta una adecuada fiabilidad, tal y como reporta en su estudio Belmonte (2013). Debe considerarse que al escoger una versión reducida del instrumento, tal y como indican los autores (Petrides, 2006), no es preceptivo su uso para valorar las dimensiones constitutivas del constructo de Autoeficacia Emocional (Bienestar Emocional, Autocontrol Emocional, Emocionabilidad y Sociabilidad), por lo que nos ha permitido obtener únicamente una valoración total del mismo. Hay que destacar que los resultados son coincidentes con los obtenidos por los autores que informan de .84 (Petrides et al., 2006); estos resultados ya habían sido corroborados previamente en muestras españolas de alumnos con el trabajo realizado por Ferrándiz, Hernández, Bermejo, Ferrando \& Sainz (2012) que reportan una fiabilidad de .82 .

El cuestionario de personalidad BFQ-NA permite evaluar desde el modelo de los Cinco Grandes la personalidad de los sujetos. Este modelo ha sido ampliamente discutido y comparado por distintos autores para esclarecer la estructura básica que mejor representa la personalidad siendo actualmente el que dispone de mayor consenso científico (Zuckerman, Kuhlman, Joireman, Teta \& Kraft, 1993; Jackson, Paunonen, Fraboni \& Coffin, 1996; Digman, 1997). Debe considerarse que, dada la importancia del modelo, el cuestionario aplicado es uno de los instrumentos más utilizados en la investigación científica y por tanto ha sido sometido a numerosas revisiones. Nuestros datos indican que dispone de una buena fiabilidad (.858); resultado similar se halló en el estudio con muestra española de Soto et al. (2011) que aportan una fiabilidad para el total de la escala de .860.

Atendiendo a nuestro objetivo de estudiarla relación entre los constructos implicados, los resultados muestran relaciones positivas, significativas y de magnitud media entre todas las dimensiones del modelo de Competencia Socio-emocional de BarOn, así como entre éstas y el nivel total de Autoeficacia Emocional. Esto aporta coherencia a los modelos de IE medidos por procedimiento auto-informado y que valoran factores más allá de la pura competencia cognitiva a nivel emocional.

La estrecha relación entre la Autoeficacia Emocional y los rasgos de personalidad del modelo de Cinco Grandes también queda constada en la investigación. Las dimensiones de la personalidad muestran relaciones positivas (a excepción del rasgo Inestabilidad Emocional) y media-alta con la Autoeficacia Emocional en consonancia con la teoría del constructo que indica que este tipo de IE (de rasgo) se compone de una disposición de rasgos y habilidades emocionales situadas en los niveles más bajos de la estructura de la personalidad. 


\section{LA RELACIÓN ENTRE LA INTELIGENCIA EMOCIONAL Y LA PERSONALIDAD EN ESTUDIANTES DE EDUCACIÓN SECUNDARIA}

Se encuentran también relaciones significativas, de signo positivo y en general de magnitud baja-media entre el modelo de Competencia Socio-emocional y los rasgos del modelo de los Cinco Grandes, exceptuando el rasgo Inestabilidad Emocional que arroja relación de signo negativo.

Los resultados también muestran relaciones significativas entre los diferentes rasgos del modeIo de Cinco Grandes menos el rasgo Inestabilidad Emocional que correlacionó de manera más baja con ellos, excepto con la Extraversión con el que no mostró correlación. Las relaciones fueron de signo positivo entre los rasgos de Conciencia, Apertura, Extraversión y Amabilidad, siendo de signo negativo la relación de éstas con la Inestabilidad Emocional y viceversa. En cuanto a la magnitud, la relación entre Conciencia y los rasgos de Apertura y Amabilidad es alta. El resto de rasgos correlacionaron con magnitudes medias, siendo la Inestabilidad Emocional la que presentó las magnitudes más bajas en su relación con los rasgos del modelo. En la misma línea, los autores del cuestionario BFQ-NA (Del Barrio, Carrasco \& Holgado, 2006) establecen relaciones entre todos los factores del mismo, mostrando en su estudio bajas magnitudes en las correlaciones del factor Inestabilidad Emocional con el resto de rasgos. Nuestros resultados son similares, no obstante nosotros no hallamos correlación significativa entre Extraversión e Inestabilidad Emocional mientras que los autores sí la hallan entre ambos rasgos, siendo la magnitud de Inestabilidad Emocional la más baja en su estudio correlacional.

A la luz de los resultados de nuestro estudio, concluimos que existe una relación manifiesta entre los constructos que componen la IE Autopercibida (IE Rasgo y Competencia Socioemocional) y los factores del modelo de personalidad de los Cinco Grandes. Por su parte, las correlaciones halladas entre los diferentes factores dan coherencia a los constructos utilizados en el estudio.

\section{REFERENCIAS}

Bar-On, R. \& Parker J. D. (2000).EQ-i:YV. Baron Emotional Quotient Inventory: Youth Version. Technical Manual. New York: MHS.

Barbaranelli, C., Caprara, G. V. \& Rabasca, A. (1998/2006). Manuale del BFQ-C. Big Five Questionnaire Children. 0.S. Organizzazioni Speciali: Firenze. (Traducción y adaptación española por Del Barrio, V; Carrasco, M.A. \& Holgado, P. Madrid: TEA, 2006).

Belmonte, V. M. (2013). Inteligencia emocional y Creatividad: Factores predictores del rendimiento académico. Tesis doctoral. Universidad de Murcia.

Bennett, G., Seashore, H. G. \&Wesman, A. G. (2000). Test de Aptitudes Diferenciales (DAT-5). Madrid: TEA

Cloninger, C.R. (1987). Neurogenetic adaptive-mechanisms in alcoholism. Science, 236, 410-416.

Costa, P.T. \& McCrae, R.R (1992).Revised NEO Personality Inventory (NEO-PI-R) and NEO Five_Factor Inventory (NEO-FFI) professional manual. Odessa, FL. Psychological Assessment Resources.

Digman, J. M. (1997). Higher order factors of the big five. Journal of Personality and Social Psychology, 73, 1246-1256.

Eysenck, H. J. (1952). The scientific study of personality. London (Ed. Cas.: Buenos Aires. Paidós, 1971).

Ferrándiz, C., Hernández, D., Bermejo, R., Ferrando, M. y Sainz, M (2012). La inteligencia emocional y social en la niñez y adolescencia: validación castellana de un instrumento para su medición. Revista de Psicodidáctica,, 17(2), 309-338.

Jackson, D. N., Paunonen, S. V., Fraboni, M. \& Goffin, R.D. (1996). A five-factor versus six factor model of personality structure. Personality and Individual Differences, 20, 33-45.

Pérez J. C., Petrides K. V., \& Furnham A. (2005).Measuring trait emotional intelligence. In Schulze 
R., Roberts R. D. (Eds.), International handbook of emotional intelligence (pp. 124-143). Cambridge, MA: Hogrefe \& Huber.

Petrides, K. V. (2006). Deriving Factor Scores from the TEIQue-SF. Webnote \#2. http://www.ioe.ac.uk/schools/phd/kpetrides/teique1.htm [17.04.2013].

Petrides, K. V., Sangareau, Y., Furnham, A., \& Frederickson, N. (2006). Trait emotional intelligence and children's peer relations at school. Social Development, 15, 537-547.

Soto, G., Ferrándiz, C., Sáinz, M., Ferrando, M., Prieto, M.D., Bermejo, R. y Hernández, D. (2011). Características psicométricas del cuestionario de personalidad BFQ-NA (Big Five Questionnaire - Niños y Adolescentes). Aula Abierta, 1, 13-24.

Yuste, C., Martínez, R. \& Galvez, J.L. (1998). Batería de aptitudes diferenciales y generales (BADyGE3). Madrid: CEPE.

Zuckerman, M., Kuhlman, D. M., Joireman, J., Teta, P. \& Kraft, M. (1993). Comparison of three structural models for personality: the big three, the big five, and the alternative five. Journal of Personality and Social Psychology, 65, 757-768. 
